\title{
ESTIMULAÇÃO ELÉTRICA NEUROMUSCULAR NA DIÁSTASE, FLACIDEZ E TROFISMO DA MUSCULATURA ABDOMINAL: UMA REVISÃO SISTEMÁTICA
}

\author{
NEUROMUSCULAR ELECTRICAL STIMULATION IN THE DIASTASIS, \\ FLACCIDITY AND TROPHISM OF THE ABDOMINAL MUSCULATURE: A \\ SYSTEMATIC REVIEW
}

\author{
Izabela Rodrigues Camilo ${ }^{1}$, Pâmela da Silva ${ }^{1}$, Tainara Buliani da Mata ${ }^{1}$, Celeide Pinto Aguiar \\ Peres $^{1}$, Gladson Ricardo Flor Bertolini ${ }^{1,2}$
}

Universidade Estadual do Oeste do Paranái ; Programa de pós-graudação em Biociências e Saúde da UNIOESTE²

\begin{abstract}
Objective: to analyze clinical trials that point to the effects of electrostimulation on abdominal diastatics, flaccidity and trophism of the abdominal musculature. Methods: A search for clinical trials was conducted in the publications accessible in the electronic databases National Library of Medicine (MEDLINE/PUbMED), Scientific Electronic Library Online (SciELO), Physiotherapy Evidence Database (PEDro) and Google academic, investigating articles related to the proposed theme, sought in the period from October to December 2017. The searches were performed using as keywords "Diastase", "Diastasis", "Eletroterapia", "Electrotherapy", "Abdominal", "Pós-parto", "Postpartum", "Flacidez" and "Flaccidity", also articulating the words with "e" or "and", thus forming combinations. Results: the electrical stimulation accompanied or not by a protocol of physical exercises in the use of abdominal muscle strengthening and/or decreased abdominal diastasis presented positive results. Conclusion: the use of Neuromuscular Electrical Stimulation is effective to decrease abdominal diastasis, flaccidity and reverse muscle hypotrophy.
\end{abstract}

Key words: Muscle Diastasis; Eletric Stimulation Therapy; Abdominal Muscles; Parturitium.

\section{Resumo}

Objetivo: analisar ensaios clínicos que apontem os efeitos da eletroestimulação na diástase abdominal, flacidez e trofismo da musculatura abdominal. Métodos: foi realizada uma busca por ensaios clínicos nas publicações acessíveis nas bases de dados eletrônicos National Library of Medicine (MEDLINE/PUbMED), Scientific Electronic Library Online (SciELO), Physiotherapy Evidence Database (PEDro) e Google acadêmico, investigando artigos relacionados ao tema proposto, procurados no período de Outubro a Dezembro de 2017. As buscas foram realizadas utilizando como palavras-chaves "Diastase", "Diastasis", "Eletroterapia", "Electrotherapy", "Abdominal", "Pós-parto", "Postpartum", "Flacidez" e "Flaccidity", articulando-se também as palavras com " $e$ " ou "and", formando assim combinações. Resultados: A estimulação elétrica acompanhada ou não de um protocolo de exercícios físicos no uso do fortalecimento da musculatura abdominal e/ou diminuição da diástase abdominal apresentou resultados positivos. Conclusão: A utilização da Estimulação Elétrica Neuromuscular é eficaz para diminuir a diástase abdominal, flacidez e reverter a hipotrofia muscular.

Palavras-chave: Diastase Muscular; Terapia por Estimulação Elétrica; Músculos Abdominais; Parto. 


\section{Introdução}

$\mathrm{Na}$ anatomia muscular da região abdominal, a única estrutura óssea presente no abdome é a da coluna lombar, de modo que órgãos da cavidade abdominal são protegidos pela musculatura da parede abdominal. Os músculos que compõem a parede abdominal são os oblíquos externo e interno, transverso do abdome, piramidal e reto abdominal. Superficialmente, os retos abdominais têm como funções flexionar a coluna, assim como servir de apoio a ela, sustentar a massa visceral e auxiliar na expiração forçada ${ }^{1,2}$

A flacidez abdominal é consequente de vários episódios ocorridos ao longo dos anos como a inatividade física, o emagrecimento demasiado, o envelhecimento, gestações, entre outros. A flacidez pode apresentar-se de duas formas distintas: a flacidez muscular e a de pele. É muito comum que os dois tipos apareçam associados, agravando o aspecto das partes do corpo afetadas pelo problema. Os músculos tornam-se flácidos principalmente pela falta de exercícios físicos e, se os mesmos não forem solicitados, as fibras tornam-se hipotrofiadas e flácidas ${ }^{3}$.

Durante o decorrer da vida de uma mulher ocorrem numerosas transformações fisiológicas. As mais acentuadas, no entanto, ocorrem durante a gestação e no período após a concepção, ou seja, no puerpério, com alterações hormonais provocadas pela relaxina, progesterona e estrógeno, associadas ao crescimento uterino, podem provocar o estiramento da musculatura abdominal, a linha da cintura pode aumentar cerca de 50 centímetros $(\mathrm{cm})$ e o músculo reto abdominal pode se alongar aproximadamente $20 \mathrm{~cm}$, podendo ocorrer uma separação na linha mediana dos músculos reto abdominais opostos, formando a diástase dos músculos retos abdominais (DMRA) e com isso há um prejuízo no vetor de forças desses músculos, ocasionando assim uma diminuição da capacidade de contração dessa musculatura ${ }^{4-7}$.

Por ser a única proteção local da região anterior do abdômen, o fortalecimento dessa musculatura tem extrema importância em casos de disfunção, buscando prevenir e tratar quaisquer deficiências. Para tanto, a eletroestimulação é um recurso utilizado tanto na recuperação quanto no tratamento da estética corporal. Ela se trata de um recurso bioelétrico que promove contrações musculares isométricas, podendo se mostrar eficaz em disfunções de fibras musculares, principalmente por conseguir ativar de 30 a $40 \%$ a mais das unidades motoras do que com exercícios comuns e em tratamentos convencionais. Existem vários relatos na literatura, que incluem resultados satisfatórios do uso da eletroestimulação para melhora da qualidade da função muscular. Os objetivos da técnica incluem: manter a qualidade e quantidade do tecido muscular, recuperar a sensação de tensão muscular, aumentar ou manter força muscular e estimular o fluxo de sangue no músculo. O aumento da força muscular com eletroestimulação pode ser alcançado em pouco tempo e este fortalecimento se dá artificialmente ${ }^{8-13}$. Contudo, ainda há conflitos na literatura que comprovem a eficácia da eletroestimulação na redução da diástase abdominal ou ganho de massa muscular na região abdominal, bem como com respeito a parâmetros e corrente utilizada. Com base nisto, o presente estudo tem como objetivo analisar ensaios clínicos que apontem os efeitos da eletroestimulação na diástase abdominal, flacidez e trofismo da musculatura abdominal.

\section{Materiais e Métodos}

Este estudo foi conduzido de acordo com critérios e recomendações Preferred Reporting Items for Systematic Reviews and Meta-Analysis (PRISMA). Para tanto, foram utilizadas referências obtidas nas bases de dados eletrônicos National Library of Medicine (MEDLINE/PubMED), Scientific Electronic Library Online (SciELO), Physiotherapy Evidence Database (PEDro) e Google acadêmico, investigando artigos relacionados ao tema proposto, procurados no período de Outubro a Dezembro de 2017. As buscas foram realizadas utilizando como palavras-chaves "Diastase", "Diastasis", "Eletroterapia", "Electrotherapy", "Abdominal", "Pós-parto", "Postpartum", "Flacidez" e "Flaccidity", articulando-se também as palavras com "e" ou "and", formando assim combinações.

Foram incluídos no estudo ensaios clínicos que tinham como objeto de estudo a influência da eletroterapia no tratamento da diástase 
abdominal (DA) e/ou flacidez e hipotrofia da musculatura abdominal, com artigos disponíveis na íntegra, online, nos idiomas português e inglês e publicados no período entre outubro de 2002 a outubro de 2017. Estudos que não se encaixassem nesses critérios foram excluídos da pesquisa bem como todos aqueles que utilizassem como terapia técnicas não existentes na prática da Fisioterapia; por utilizarem a eletroacupuntura e que fossem revisão da literatura.

Os estudos tiveram seu conteúdo analisado por três avaliadores de forma independente e cega, sendo esta realizada individualmente, selecionados por consenso aqueles que atendessem aos critérios de inclusão, se o consenso entre estes não fosse alcançado, um quarto examinador seria consultado. A sequência de etapas para seleção dos artigos foi feita primeiramente pela identificação e seleção de artigos pelo título, seguido da elegibilidade em que se avaliou o resumo do artigo e em seguida se aplicavam os critérios de inclusão e exclusão, e por fim a inclusão dos artigos escolhidos. Para os ensaios clínicos randomizados foi utilizada a Escala de Jadad como forma avaliar sua qualidade ${ }^{14}$.

\section{Resultados}

Após a pesquisa nas bases de dados foi encontrado um número total de 1675 artigos científicos, sendo 1223 artigos encontrados no MEDLINE/PubMED, 64 no PEDro, 46 no SciELO e 342 no Google Acadêmico. Seguindo essa etapa, ocorreu a análise de títulos, na qual foram selecionados apenas 19 artigos e por fim, após a análise criteriosa dos artigos, chegou-se a um total de quatro artigos selecionados para esta revisão sistemática (figura 1 ).

Os resultados obtidos da análise dos artigos incluídos neste estudo mostraram que a estimulação elétrica acompanhada ou não de um protocolo de exercícios físicos no uso do fortalecimento da musculatura abdominal e/ou diminuição da diástase abdominal apresenta resultados positivos (quadro 1 ).

Figura 1: Fluxograma para identificação dos artigos incluídos no estudo

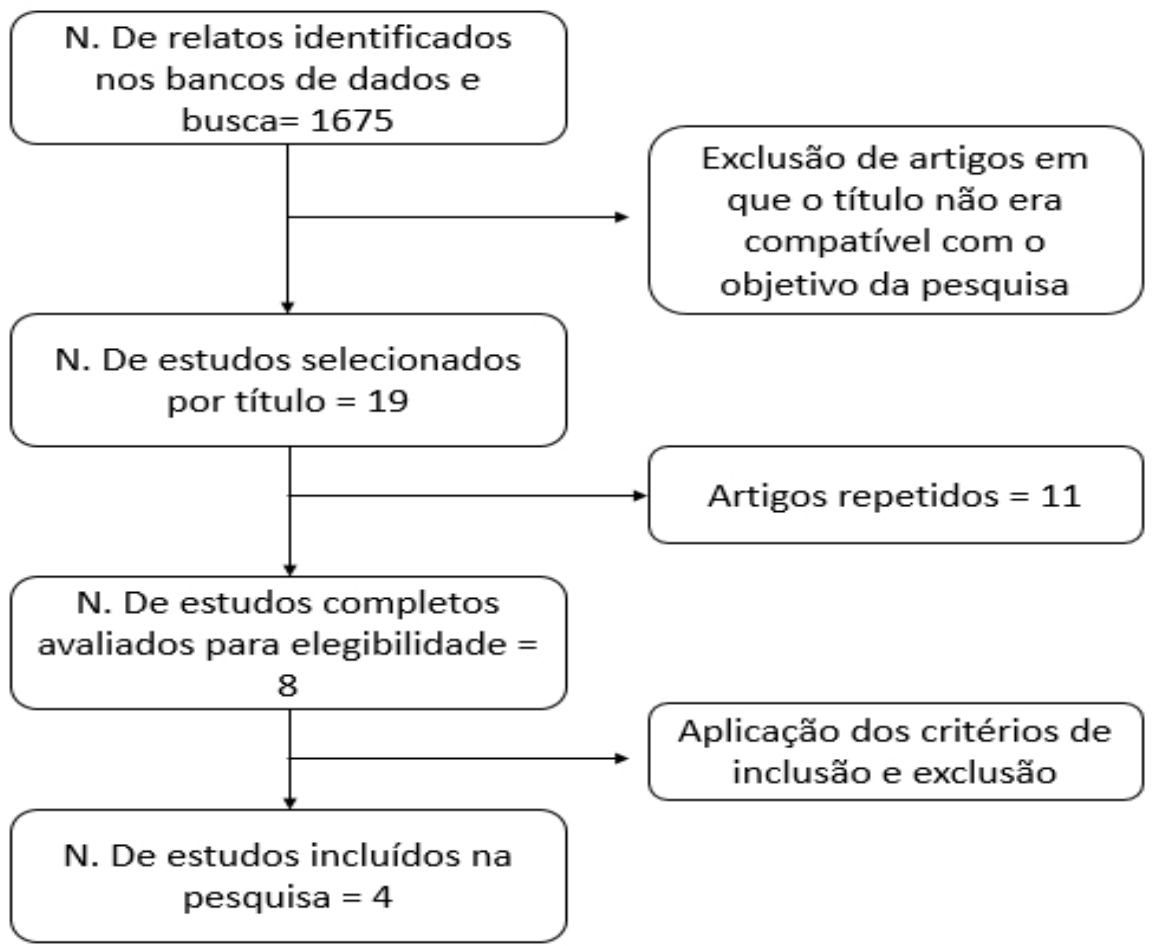


Quadro 1: Estudos incluídos na revisão sistemática abordando eletroterapia nas disfunções do músculo reto abdominal.

\begin{tabular}{|c|c|c|c|c|c|c|}
\hline $\begin{array}{l}\text { Autor, } \\
\text { Ano (País) }\end{array}$ & Objetivo & $\begin{array}{l}\text { Tipo de } \\
\text { Estudo }\end{array}$ & Amostra & Protocolo & Resultados & Jadad \\
\hline $\begin{array}{l}\text { Borges e } \\
\text { Valentin } \\
15, \quad 2002 \\
\text { (Brasil). }\end{array}$ & $\begin{array}{lr}\text { Descrição de } & \text { de } \\
\text { casos de } & 3 \\
\text { pacientes } & \text { no } \\
\text { puerpério, com } \\
\text { diástase } \\
\text { abdominal } \\
\text { tratadas } \\
\text { EENM. }\end{array}$ & $\begin{array}{l}\text { Estudo } \\
\text { caso. }\end{array}$ & $\begin{array}{l}\text { Três pacientes } \\
\text { em puerpério } \\
\text { de parto } \\
\text { normal. }\end{array}$ & $\begin{array}{l}\text { EENM por } 20 \\
\text { minutos, } 3 \\
\text { vezes } \\
\text { semana, } \\
\text { durante seis } \\
\text { semanas. }\end{array}$ & $\begin{array}{l}\text { Redução da } \\
\text { diástase } \\
\text { abdominal num } \\
\text { tempo menor que } \\
\text { o fisiológico e } \\
\text { pôde-se reduzir } \\
\text { medida pelo } \\
\text { encurtamento do } \\
\text { reto abdominal. }\end{array}$ & 0 \\
\hline $\begin{array}{ll}\text { Kamel } & \text { e } \\
\text { Yousif, } & \\
2017 & 16 \\
\text { (Egito) } & \end{array}$ & $\begin{array}{ll}\text { Avaliação } & \text { da } \\
\text { EENM na } & \text { na } \\
\text { recuperação da } & \text { força muscular } \\
\text { abdominal em } \\
\text { mulheres com } \\
\text { diástase de reto } \\
\text { abdominal. }\end{array}$ & $\begin{array}{l}\text { Estudo de } \\
\text { controle } \\
\text { randomizado. }\end{array}$ & $\begin{array}{ll}60 \quad \text { mulheres } \\
\text { puérperas, } \\
\text { meses após o } \\
\text { parto. }\end{array}$ & $\begin{array}{l}\text { Exercícios } \\
\text { abdominais e } \\
\text { EENM durante } \\
8 \text { semanas. }\end{array}$ & $\begin{array}{l}\text { A adição da } \\
\text { estimulação } \\
\text { elétrica } \\
\text { neuromuscular ao } \\
\text { programa de } \\
\text { reabilitação DRAM } \\
\text { mostrou } \\
\text { resultados } \\
\text { positivos para a } \\
\text { recuperação de } \\
\text { músculos } \\
\text { abdominais após o } \\
\text { parto. }\end{array}$ & 4 \\
\hline 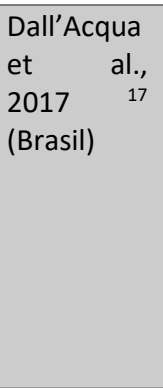 & $\begin{array}{lr}\text { Avaliar } & \text { e } \\
\text { comparar } & \text { os } \\
\text { efeitos da } & \text { EENM } \\
\text { associada a } & \\
\text { tratamento } & \\
\text { fisioterapêutico } \\
\text { em doentes } \\
\text { críticos. }\end{array}$ & $\begin{array}{l}\text { Estudo de } \\
\text { controle } \\
\text { randomizado } \\
\text { duplo-cego. }\end{array}$ & $\begin{array}{l}25 \text { pacientes } \\
\text { hospitalizados } \\
\text { em uma } \\
\text { unidade de } \\
\text { terapia } \\
\text { intensiva. }\end{array}$ & $\begin{array}{l}\text { EENM e } \\
\text { fisioterapia } \\
\text { motora } \\
\text { convencional. }\end{array}$ & $\begin{array}{l}\text { Houve diferença } \\
\text { estatisticamente } \\
\text { significativa entre } \\
\text { a intervenção e os } \\
\text { grupos } \\
\text { convencionais na } \\
\text { espessura do } \\
\text { músculo } \\
\text { abdominal } \\
\text { peitoral. }\end{array}$ & 5 \\
\hline $\begin{array}{l}\text { Klefens et } \\
\text { al., } 2013{ }^{18} \\
\text { (Brasil). }\end{array}$ & $\begin{array}{lr}\begin{array}{l}\text { Verificar } \\
\text { redução }\end{array} & \text { do } \\
\text { perímetro } & \\
\text { abdominal } & \text { em } \\
\text { voluntária } & \text { pós- } \\
\text { gestacional, com } & \\
\text { diástase do } & \text { reto } \\
\text { abdominal, } \\
\text { submetida } \\
\text { EENM a }\end{array}$ & $\begin{array}{l}\text { Relato } \\
\text { caso. }\end{array}$ & $\begin{array}{l}\text { Uma } \\
\text { participante do } \\
\text { sexo feminino } \\
\text { com quadro de } \\
\text { diástase de } \\
\text { reto abdominal } \\
\text { pós- } \\
\text { gestacional. }\end{array}$ & $\begin{array}{l}10 \text { sessões de } \\
\text { EENM. }\end{array}$ & $\begin{array}{l}\text { A EENM trouxe } \\
\text { benefícios no que } \\
\text { se refere à } \\
\text { diminuição } \\
\text { DMRA. }\end{array}$ & 0 \\
\hline
\end{tabular}

Legenda: DMRA - Diástase do músculo reto abdominal; EENM - Estimulação Elétrica Neuromuscular 


\section{Discussão}

Diante dos efeitos deletérios que as disfunções da musculatura abdominal proporcionam tanto na estética quanto na funcionalidade dos indivíduos, faz-se necessário estudos sobre protocolos de tratamento para tais casos. Dentre os recursos disponíveis na pratica terapêutica, encontra-se a eletroestimulação para melhora da qualidade da função muscular. Neste estudo foram encontrados 4 ensaios clínicos com efeitos positivos para o tratamento das disfunções da musculatura abdominal ${ }^{15-18}$.

No estudo de Borges e Valentin ${ }^{15}$, foi utilizada eletroestimulação neuromuscular de média frequência, com frequência portadora de $2.500 \mathrm{~Hz}$, frequência modulada de $100 \mathrm{~Hz}$, fase (ciclo) de 50\%, tempo de contração de $6 \mathrm{seg}$. e tempo de repouso de 6 seg., por um tempo total de estimulação de 20 minutos, numa intensidade (mA) confortável e suficiente para proporcionar contração visível, três vezes por semana, durante seis semanas. Após a intervenção foi evidenciado através do uso de perimetria, fotos e o uso de paquímetro clara redução da diástase num período menor que o fisiológico. Os autores sugerem a importância de se analisar o quadro clínico puerperal com o objetivo de planejar e implementar o tratamento adequado visando uma recuperação mais rápida em relação ao tempo normal de restabelecimento pós-parto.

Kamel e Yousif 16 associaram à estimulação elétrica neuromuscular (corrente de saída máxima de $34 \pm 5 \quad \mathrm{~mA}, 500 \mathrm{~W}$, largura de pulso de 100-600 $\mu \mathrm{S}$ e taxa de pulso de 1-500 pulsos/s) um protocolo de exercícios para fortalecimento da musculatura abdominal no tratamento da diástase, três vezes por semana durante oito semanas, evidenciando que tanto o exercício físico isolado quanto associado à estimulação elétrica neuromuscular tiveram uma diminuição significativa da DMRA, porém o grupo que associou ambas as terapias, teve uma diminuição e ganho de força consideravelmente maior.

No trabalho de Klefens et al. ${ }^{18}$, foi utilizado um protocolo de EENM, com frequência portadora de $2.500 \mathrm{~Hz}$, modulada em $100 \mathrm{~Hz}$, ciclo de $50 \%$, tempo rise de 1 segundo, ON de 6 segundos, decay de 1 segundo, e OFF de 6 segundos, com intensidade $(\mathrm{mA})$ regulada de acordo com a sensibilidade da participante e que provocou contração muscular visível, com sessões de 20 minutos, duas vezes por semana, durante cinco semanas. Após a intervenção, foi evidenciado diminuição da DMRA. Os autores implicam que devido à fraqueza dos músculos retos abdominais no pós-gestacional sua reabilitação precoce é de grande valia, principalmente para que a mulher retorne o mais rápido e em boas condições físicas às suas atividades de vida diária. Ressaltam ainda que a EENM é mais eficaz que a contração voluntária, embora a literatura relate que seu uso juntamente com a contração voluntária enobreça o tratamento.

Em outro estudo, Dall'Acqua et al. ${ }^{17}$ avaliaram a influência da eletroestimulação neuromuscular (duração do pulso $300 \mu \mathrm{s}$, tempo de subida 1 segundo, tempo de estímulo (ON) 3 segundos, tempo de decaimento 1 segundo e tempo de relaxamento (OFF) $10 \mathrm{~s}$ ) associada a exercícios fisioterapêuticos convencionais no aumento da espessura da musculatura abdominal e peitoral em pacientes que haviam sido hospitalizados há mais de 15 dias e tinham recebido pelo menos $24 \mathrm{~h}$ de VMI, mostrou diferença estatisticamente significativa na espessura do músculo abdominal e peitoral entre os grupos intervenção e tratamento convencional. $\mathrm{Na}$ comparação entre a avaliação inicial e a avaliação final em cada grupo, não houve alteração na massa muscular no grupo de intervenção, enquanto houve uma diminuição dessas medidas no grupo tratamento convencional. $E$ ainda os pacientes que receberam a intervenção permaneceram por menos tempo internados na UTI. Os autores sugerem que o NMES pode ter um efeito protetor contra a perda muscular.

O uso da Estimulação Elétrica Neuromuscular no tratamento das disfunções da musculatura abdominal pode ser utilizado para minimizar ou prevenir tais acometimentos, porém, não há padrões definidos na literatura quanto aos parâmetros da corrente e duração da utilização. Ensaios clínicos sobre o assunto são escassos na literatura e os encontrados evidenciam divergências e precariedades em sua metodologia, porém, em suma, a EENM 
apresenta efeitos positivos no tratamento da diástase, flacidez e manutenção do trofismo da musculatura abdominal quando utilizada em médias frequências e associada ou não a exercícios físicos.

\section{Conclusão}

Com base nos estudos analisados, a utilização de Estimulação Elétrica Neuromuscular é eficaz para diminuir a diástase e flacidez abdominal, sendo também eficaz na reversão da hipotrofia muscular. Foi observado que ao associar a estimulação elétrica com exercícios físicos, os resultados são ainda mais satisfatórios. Entretanto há necessidade de realizar novos ensaios clínicos randomizados, que possibilitem uma maior discussão e reforço dos efeitos dessa terapia, visto que ainda é pequeno número de estudos dificultando a realização de análises mais aprofundadas, como meta-análises.

\section{Referências}

1. Benatti AT. Equilíbrio tóraco-abdominal: ação integrada à respiração e à postura. Arq Ciências da Saúde da UNIPAR. 2001;5(1):87-92.

2. Gouveia KMC, Gouveia EC. O músculo transverso abdominal e sua função de estabilização da coluna lombar. Fisioter em Mov. 2008;21(3):45-50.

3. Lima PEF, Rodrigues GBDO. A estimulação russa no fortalecimento da musculatura abdominal / Russian stimulation in strengthening abdominal muscle. ABCD Arq Bras Cir Dig. 2012;25(2):125-8.

4. Souza VB de, Roecker S, Marcon SS. Ações educativas durante a assistência pré-natal: percepção de gestantes atendidas na rede básica de Maringá-PR. Rev Eletrônica Enferm. 2011;13(2):199-210.

5. Costa ES, Pinon GMB, Costa TS io, Santos RC de A, Nóbrega AR, Sousa LB de. Physiological Alterations From the Pespective of Women in Pregnancy. Rev da Rede Enferm do Nord. 2010;11:86-93.

6. Strapasson MR, Nedel MNB. Puerpério imediato: desvendando o significado da maternidade. Rev Gaúcha Enferm. 2010;31(3):521-8.

7. Mesquita LA, Machado AV, Andrade AV. Fisioterapia para redução da diástase dos músculos retos abdominais no pós-parto. Rev Bras Ginecol e Obs. 1999;21(5):267-72.

8. Hicks GE, Sions MJ, Velasco TO, Manal TJ. Trunk muscle training augmented with neuromuscular electrical stimulation appears to improve function in older adults with chronic low back pain: a randomized preliminary trial. Clin J Pain. 2016;32(10):898-906.

9. Jones S, Man WDC, Gao W, Higginson IJ, Wilcock A, Maddocks M. Neuromuscular electrical stimulation for muscle weakness in adults with advanced disease (Review). Cochrane Libr. 2016;(10).

10. Hauger A V., Reiman MP, Bjordal JM, Sheets C, Ledbetter L, Goode AP. Neuromuscular electrical stimulation is effective in strengthening the quadriceps muscle after anterior cruciate ligament surgery. Knee Surgery, Sport Traumatol Arthrosc. 2018;26(2):399-410.

11. Coghlan S, Crowe L, Mccarthypersson U, Minogue C, Caulfield B. Neuromuscular electrical stimulation training results in enhanced activation of spinal stabilizing muscles during spinal loading and improvements in pain ratings. Conf Proceedings 33rd Annu Int Conf IEEE EMBS. 2011;30(3):7622-5.

12. Chen RC, Li XY, Guan LL, Guo BP, Wu WL, Zhou ZQ, et al. Effectiveness of neuromuscular electrical stimulation for the rehabilitation of moderate-to-severe COPD: A meta-analysis. Int J COPD. 2016;11(1):2965-75.

13. Kim SY, Kim JH, Jung GS, Baek SO, Jones $\mathrm{R}$, Ahn SH. The effects of transcutaneous neuromuscular electrical stimulation on the activation of deep lumbar stabilizing muscles of patients with lumbar degenerative kyphosis. J Phys Ther Sci. 2016;28(2):399-406.

14. Malavolta EA, Gobbi RG, Mancuso Filho $J A$, Demange MK. Análise crítica das publicações científicas da Revista Brasileira de Ortopedia no período de 2006 a 2010. Rev Bras Ortop. 2013;48(3):211-5.

15. Borges F dos S, Valentin EC. Tratamento da flacidez e diástase do reto-abdominal no puerpério de parto normal com o uso de eletroestimulação muscular com corrente de média frequência - estudo de caso. Rev Bras Fisioter Dermato-Funcional. 2002;1(1):1-9.

16. Kamel DM, Yousif AM. Neuromuscular electrical stimulation and strength recovery of postnatal diastasis recti abdominis muscles. Ann Rehabil Med. 2017;41(3):465-72. 
17. Dall'Acqua AM, Sachetti A, Santos LJ, Lemos FA, Bianchi T, Naue WS, et al. Use of neuromuscular electrical stimulation to preserve the thickness of abdominal and chest muscles of critically ill patients: A randomized clinical trial. J Rehabil Med. 2017;49(1):40-8.

18. Klefens SO, Deon KC, Medeiros T. Uso da estimulação elétrica neuromuscular no manejo da diástase de reto abdominal pós-gestacional: relato de caso. Rev Uniandrade. 2013;14(3):2419.

\section{Endereço para Correspondência}

Rua Universitária, 2069. Jardim Universitário

Cascavel - PR

CEP.: 85.819-110

gladsonricardo@gmail.com

Recebido em 28/11/2019

Aprovado em 14/02/2020

Publicado em 05/02/2021 